\title{
Intrinsic Kinetic Limitations in Substituted Lithium Layered Transition-Metal Oxide Electrodes
}

Antonin Grenier, ${ }^{\dagger, \perp}$ Philip J. Reeves, ${ }^{\ddagger}$ Hao Liu, ${ }^{\dagger}$ Ieuan D. Seymour, ${ }^{\ddagger}$ Katharina Märker, ${ }^{\ddagger}$ Kamila M. Wiaderek, ${ }^{\dagger}$

Peter J. Chupas, ${ }^{\perp}$ Clare P. Grey, ${ }^{\ddagger}$ Karena W. Chapman ${ }^{*}+, \perp$

${ }^{+} X$-ray Science Division, Advanced Photon Source, Argonne National Laboratory, 9700 South Cass Avenue, Lemont, Illinois 60439, United States

${ }^{\perp}$ Department of Chemistry, Stony Brook University, 100 Nicolls Road, New York 11794, United States

‡Department of Chemistry, University of Cambridge, Lensfield Road, Cambridge, CB2 1EW, United Kingdom

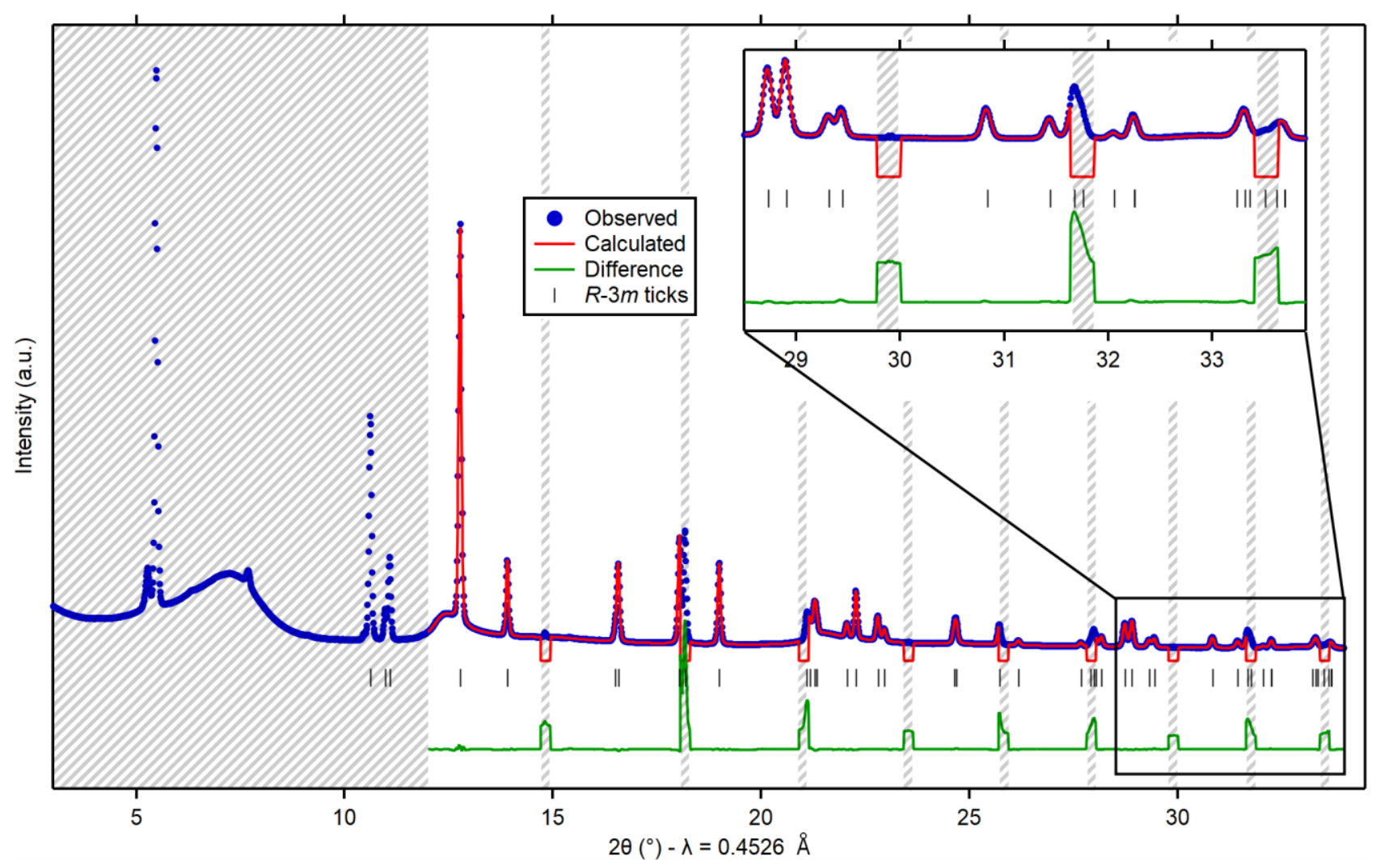

Figure S1. Typical Rietveld refinement to the synchrotron operando XRD data (here, first frame, time $=0 \mathrm{~h}$ ) presented in Figure 3 of the main text. The dashed areas represent $2 \theta$ regions excluded from the refinement, as explained in the experimental section. Refined interlayer mixing $\boldsymbol{m}=\mathbf{0 . 0 1 8 8 ( 4 ) \text { . }}$ 
Table S1. First cycle specific capacities (Q) and Coulombic efficiencies (CE) for the electrochemical measurements of NCA and LCO shown Figure 1 of the main text. Note, the measured capacities were not corrected for the residual current typically observed during the potentiostatic (PS) hold (cf. Figures S2 and S3).

\begin{tabular}{lcccc}
\hline & \multicolumn{3}{c}{ NCA Toda } & LCO Sigma-Aldrich \\
\hline Charge cut-off voltage (V) & 4.5 & 4.1 & 3.7 & 4.1 \\
\hline Charge Q $\left(\mathrm{mAh} \cdot \mathrm{g}^{-1}\right.$ ) & 229.3 & 180.5 & 60.8 & 122.7 \\
Discharge Q $\left(\mathrm{mAh} \cdot \mathrm{g}^{-1}\right)$ & 204.0 & 157.9 & 30.0 & 120.3 \\
Apparent Q loss $\left(\mathrm{mAh} \cdot \mathrm{g}^{-1}\right)$ & 25.3 & 22.6 & 30.8 & 2.4 \\
CE (\%) & 89.0 & 87.5 & 49.3 & 98.1 \\
\hline PS hold Q (mAh. $\left.{ }^{-1}\right)$ & 20.2 & 20.3 & 27.1 & 1.5 \\
Irreversible Q loss (mAh. $\left.\mathrm{g}^{-1}\right)$ & 5.1 & 2.3 & 3.7 & 0.9 \\
CE w/ 24 h PS hold (\%) & 97.8 & 98.7 & 93.8 & 99.3 \\
\hline
\end{tabular}

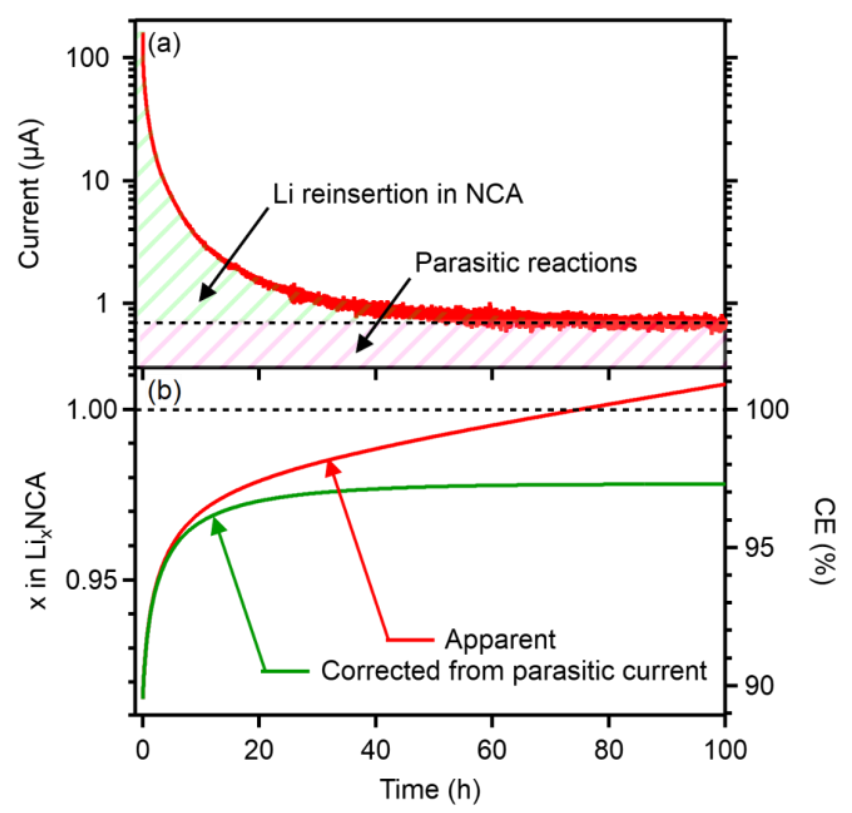

Figure S2. (a) Evolution of the current for an NCA electrode subjected to a $100 \mathrm{~h}$ potentiostatic discharge hold at $2.7 \mathrm{~V}$, after a galvanostatic charge-discharge between 4.5 and $2.7 \mathrm{~V}$ at C/15. Contributions attributed to Li reinsertion in NCA and parasitic reactions are illustrated by the green and purple hatched areas, respectively. The steady-state current of $0.7 \mu \mathrm{A}$ is ascribed to parasitic reactions based on measurements performed on a blank electrode (without NCA) shown Figure S2. (b) The Coulombic efficiency and calculated Li concentration in NCA during the potentiostatic discharge, with or without correction for parasitic reactions (assuming constant 0.7 $\mu A$ current throughout the hold). 


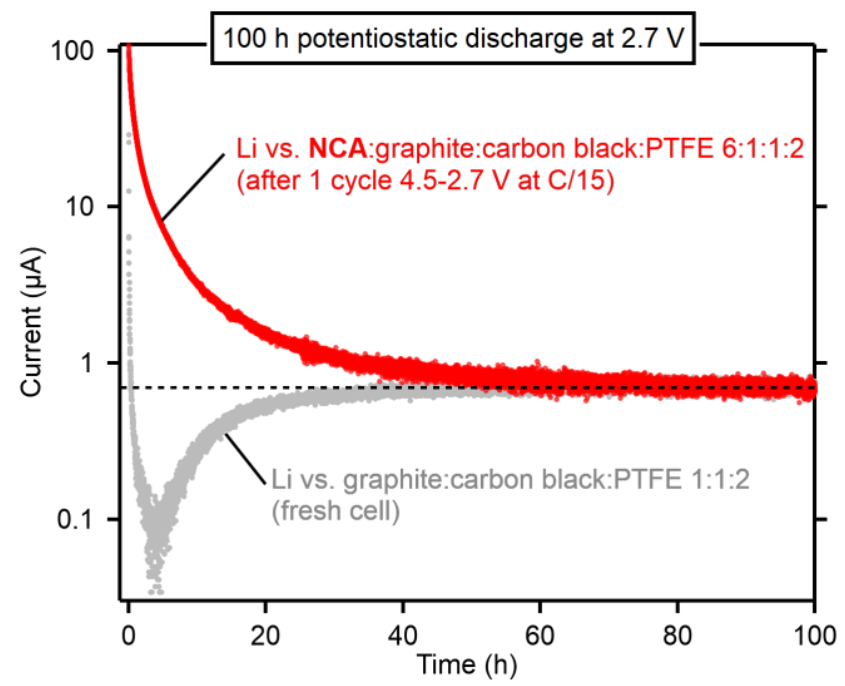

Figure S3. Evolution of the current over a $100 \mathrm{~h}$ potentiostatic discharge step at $2.7 \mathrm{~V}$ for a blank electrode containing only electrode additives, i.e., carbon black, graphite and PTFE. The steady-state residual current of $0.7 \mu \mathrm{A}$ matches the residual current measured for a cycled NCA electrode subjected to a similar treatment (Figure S1). This shows that the residual current is not related to Li reinsertion in NCA, but is due to parasitic side reactions involving other cell components. 

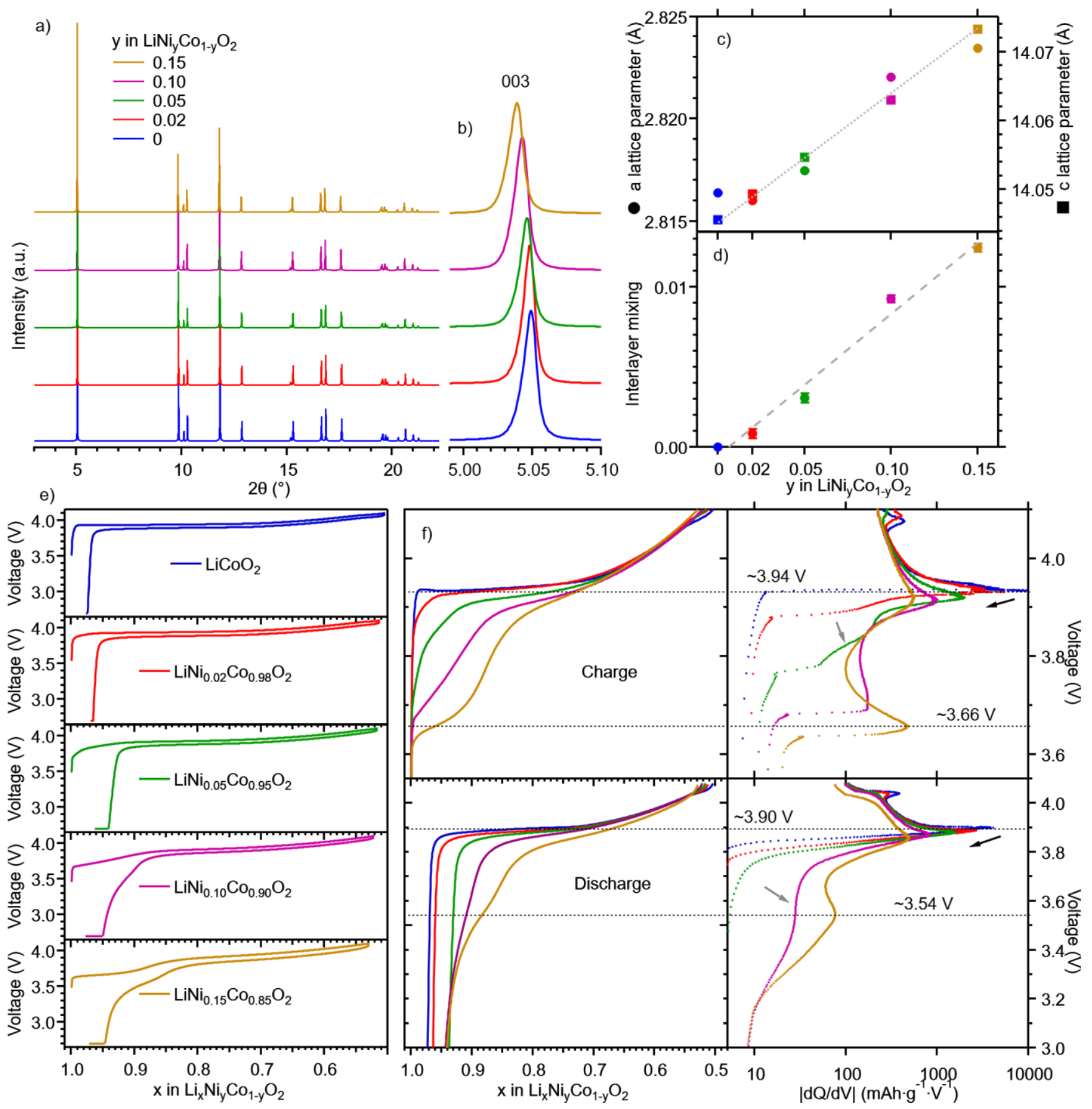

Figure S4. (a) High-resolution XRD patterns $(\lambda=0.412682 A)$ recorded on $\mathrm{LiNi}_{y} \mathrm{Co}_{1-y} \mathrm{O}_{2}$ powders. All peaks are indexed by the $R-3 m$ space group. (b) Zoom in on the 003 reflection showing how Ni incorporation induces a shift. (c) $a$ and $c$ lattice parameters and (d) interlayer mixing obtained from Rietveld refinements. Dashed lines are guides for the eyes. (e) Electrochemical profiles (4.1 V - $2.7 \mathrm{~V}$ at $\mathrm{C} / 15$ and $24 \mathrm{~h}$ potentiostatic hold at $2.7 \mathrm{~V}$ ) recorded on $\mathrm{LiNi}_{\mathrm{y}} \mathrm{Co}_{1-\mathrm{y}} \mathrm{O}_{2}$ electrodes.(f) Overlaid charge/discharge profiles and with corresponding differential capacity plots.

Near-linear increase of lattice parameters with increasing Ni doping suggest successful incorporation of $\mathrm{Ni}$ in the layered structure [ionic radius of low-spin $\mathrm{Ni}^{3+}(0.56 \AA)$ is larger than that of $\mathrm{Co}^{3+}(0.545 \AA)$ ], consistent with previous reports. ${ }^{1}$ The additional low-voltage step, which lengthens with increasing Ni doping, is also consistent with previous reports, and further suggests successful incorporation of Ni. The arrows on dQ/dV plots show how peaks shift and vary as $y$ increases. These peaks may be attributed to preferential Co $(\sim 3.90-3.94 \mathrm{~V})$ and $\mathrm{Ni}(3.54-3.66 \mathrm{~V})$ redox. ${ }^{2}$ The larger irreversible capacity loss (i.e., after potentiostatic hold) observed for as prepared $\mathrm{LiCoO}_{2}(6.5 \mathrm{mAh} / \mathrm{g})$, compared with commercial $\mathrm{LiCoO}_{2}(0.9 \mathrm{mAh} / \mathrm{g})$, may be explained by one or several of the following factors: 
- $\quad$ Structural defects such as stacking faults and/or dislocations preventing reinsertion in specific Li sites. The presence of defects along the $c$ axis is suggested from the asymmetry of $00 l$ Bragg peaks. In that regard, the reported lattice parameters, including those of $\mathrm{Ni}$ doped LCO, reflect an average of the distribution of lattice parameters induced by these defects.

- Increased amount of surface side reactions (e.g., Co dissolution) in the as prepared sample due its smaller crystallite size-a $~ 200$ $\mathrm{nm}$ crystallite size is estimated from the XRD data (using the LVol_FWHM_CS_G_L macro implemented in TOPAS), while the commercial LCO sample is micrometer-sized, according to the manufacturer's specifications.

A comparable irreversible capacity loss is observed for all compositions. Its presence does not challenge the fact that increasing Ni content leads to additional capacity recovered during potentiostatic hold, i.e., kinetic limitation.
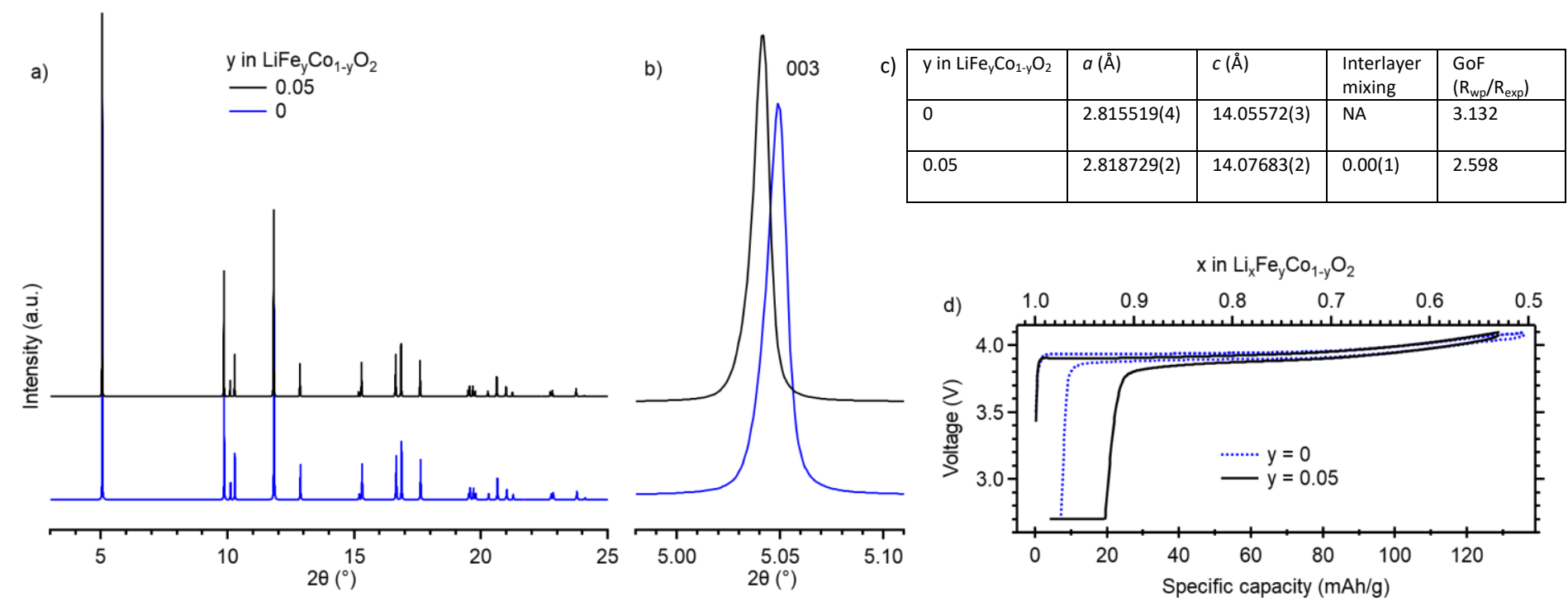

Figure S5. (a) High-resolution XRD patterns $(\lambda=0.412682 A)$ recorded on $\mathrm{LiFe}_{\mathrm{y}} \mathrm{Co}_{1-\mathrm{y}} \mathrm{O}_{2}$ powders. All peaks are indexed by the $R-3 m$ space group. (b) Zoom in on the 003 reflection. (c) a and $c$ lattice parameters, interlayer mixing (Fe-Li exchange) and goodness of fit obtained for the refinements. (d) Electrochemical profile $(4.1 \mathrm{~V}-2.7 \mathrm{~V}$ at $\mathrm{C} / 15$ and $24 \mathrm{~h}$ potentiostatic hold at $2.7 \mathrm{~V})$ recorded on LiFe ${ }_{0.05} \mathrm{Co}_{0.95} \mathrm{O}_{2}$ electrode. The profile of as prepared $\mathrm{LiCoO}_{2}$ is overlaid for comparison. The preparation of the material is detailed in the main text.

Similar to the Ni-doped analogue, the successful incorporation of $\mathrm{Fe}^{3+}$ in the structure can be inferred from the increase in lattice parameters (high-spin $\mathrm{Fe}^{3+}$ has an ionic radius of $0.645 \AA$ ) and the change in the electrochemical profile, consistent with previous reports. ${ }^{3}$ No sign of $\alpha-$ $\mathrm{LiFeO}_{2}$ was observed, unlike previously reported for $2 \% \mathrm{Fe}$ nominal doping attempts, ${ }^{4}$ perhaps due to the use of nanoscale precursor or Unlike with $\mathrm{Ni}$, Fe doping does not generate an extra voltage step, suggesting that $\mathrm{Co}$ and Fe redox occurs concomitantly, as previously reported. ${ }^{3}$ 
Table S2. First cycle specific capacities (Q) and Coulombic efficiencies (CE) for the electrochemical measurements of Ni and Fe doped LCO shown Figures S3 and S4. Note, the measured capacities were not corrected for the residual current typically observed during the potentiostatic step (Figures S2).

\begin{tabular}{|c|c|c|c|c|c|c|c|}
\hline$y$ in $\mathrm{Li}(\mathrm{Ni}, \mathrm{Fe})_{\mathrm{y}} \mathrm{Co}_{1-\mathrm{y}} \mathrm{O}_{2}$ & 0.00 & $0.02 \mathrm{Ni}$ & $0.05 \mathrm{Ni}$ & $0.10 \mathrm{Ni}$ & $0.15 \mathrm{Ni}$ & $0.05 \mathrm{Fe}$ & $\begin{array}{c}\text { LCO Sigma- } \\
\text { Aldrich }\end{array}$ \\
\hline Discharge $Q\left(m A h \cdot g^{-1}\right)$ & 128.8 & 124.1 & 116.4 & 117.4 & 114.4 & 109.7 & 120.3 \\
\hline Apparent $Q$ loss (mAh. $\left.g^{-1}\right)$ & 7.1 & 9.5 & 16.2 & 13.7 & 14.7 & 19.4 & 2.4 \\
\hline CE (\%) & 94.8 & 92.9 & 87.8 & 89.6 & 88.6 & 85.0 & 98.1 \\
\hline Irreversible $Q$ loss (mAh. $\mathrm{g}^{-1}$ ) & 6.5 & 8.4 & 10.3 & 6.2 & 7.8 & 4.1 & 0.9 \\
\hline CE w/ 24 h PS hold (\%) & 95.2 & 93.7 & 92.2 & 95.3 & 93.9 & 96.9 & 99.3 \\
\hline
\end{tabular}

a

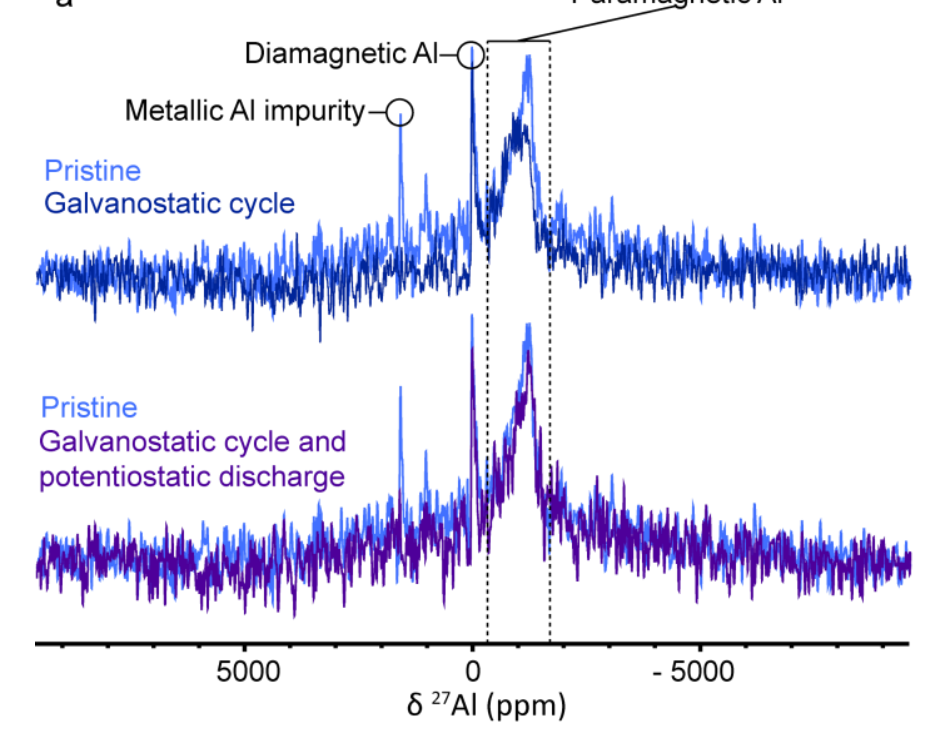

Paramagnetic A

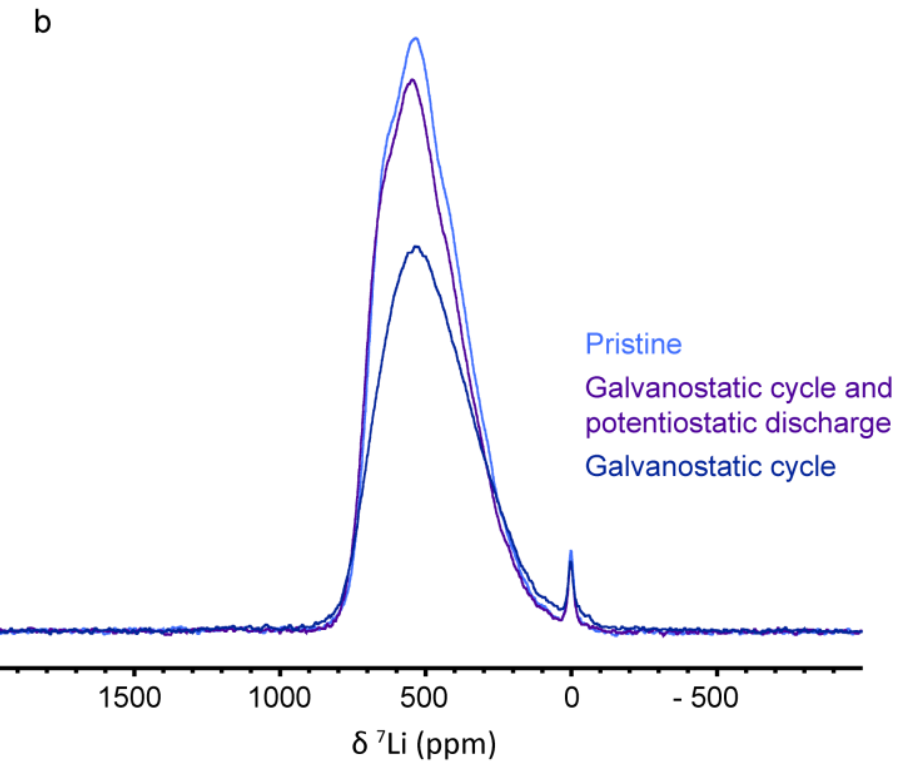

Figure S6. (a) ${ }^{27}$ Al NMR spectra for pristine, galvanostatically cycled and galvanostatically cycled with potentiostatic discharge step samples of NCA. Following the assignments made in previous work, ${ }^{5}$ signal intensity at the most highly shifted end of the paramagnetic $\mathrm{Al}$ region highlights that in the sample that is only galvanostatically discharged, the $\mathrm{Ni}^{4+}$ is not as fully reduced to $\mathrm{Ni}^{3+}$ as in the potentiostatically discharged sample. (b) pj-MATPASS NMR spectrum of the same samples. pj-MATPASS suppresses the spinning sidebands, highlighting that there are no additional resonances present. For example, any $\mathrm{Li}_{\text {in }}$ the mainly $\mathrm{Ni}^{3+}$ transition metal layer would be expected to have a small negative shift; its only paramagnetic neighbors are $90^{\circ}$ interactions from $\mathrm{Ni}^{3+}$ which, due to a $t_{2 g}-p_{\pi}-s$ polarization mechanism, should give rise to small negative shifts ${ }^{6}$. Such resonances are not observed. 


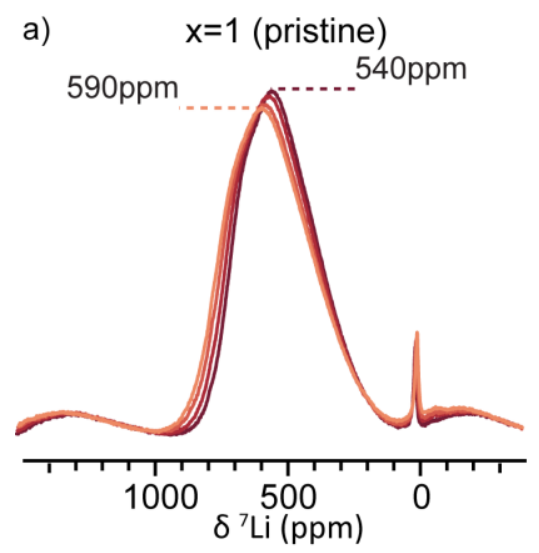

d)

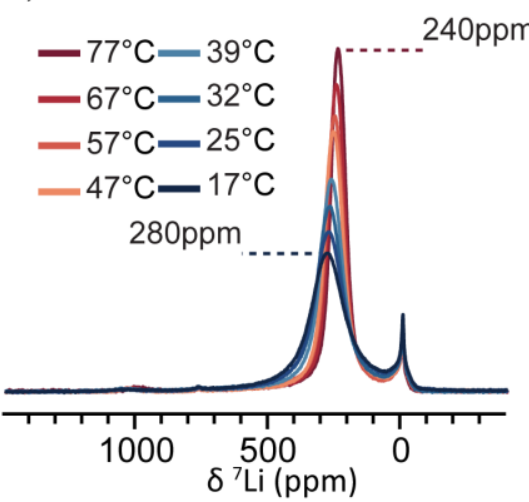

b)

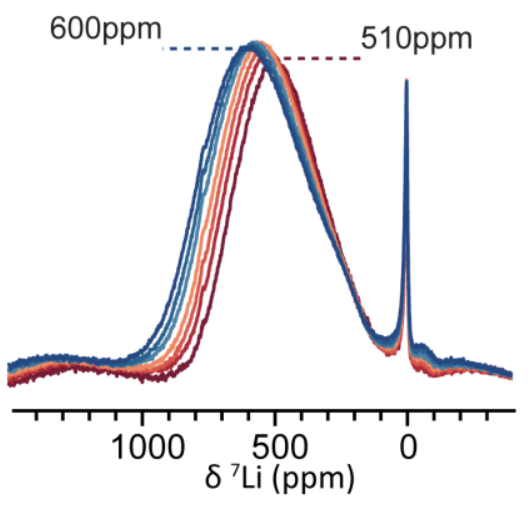

e)

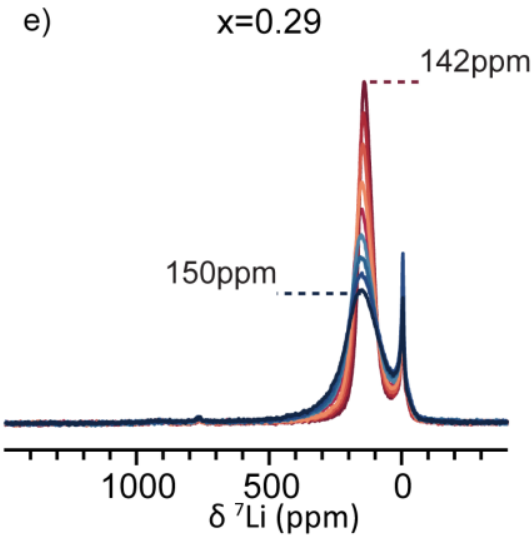

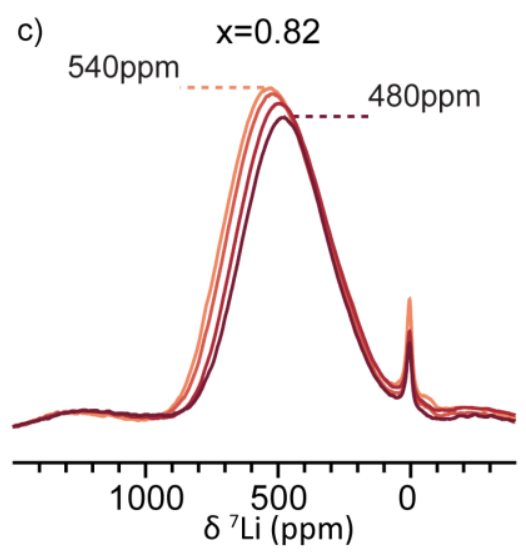

f)
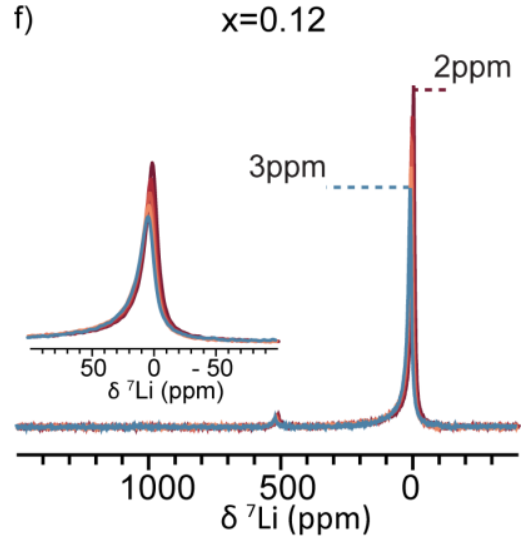

Figure S7. ${ }^{7} \mathrm{Li}$ spectra of NCA at various states of charge and temperatures. $x$ denotes the amount of Li remaining per formula unit calculated from the electrochemistry. a) and b) remain relatively unchanged by temperature variation. c),d),e) and f) have sharper lineshapes at higher temperature indicating the Li motion is enhanced. All the shifts (which are caused by the paramagnetic Fermi-contact interaction) are expected to have a Curie-Weiss like dependence hence the resonances move to lower shifts at higher temperatures in all the samples. Within each panel, spectra are displayed without scaling, between panels the spectra are normalised arbitrarily so that the most intense spectra all have the same maximum intensity for ease of comparison. Spectra were recorded using Hahn-echo pulse sequences at $60 \mathrm{kHz}$ using a Bruker 1.3mm MAS probe at 4.7 T. 

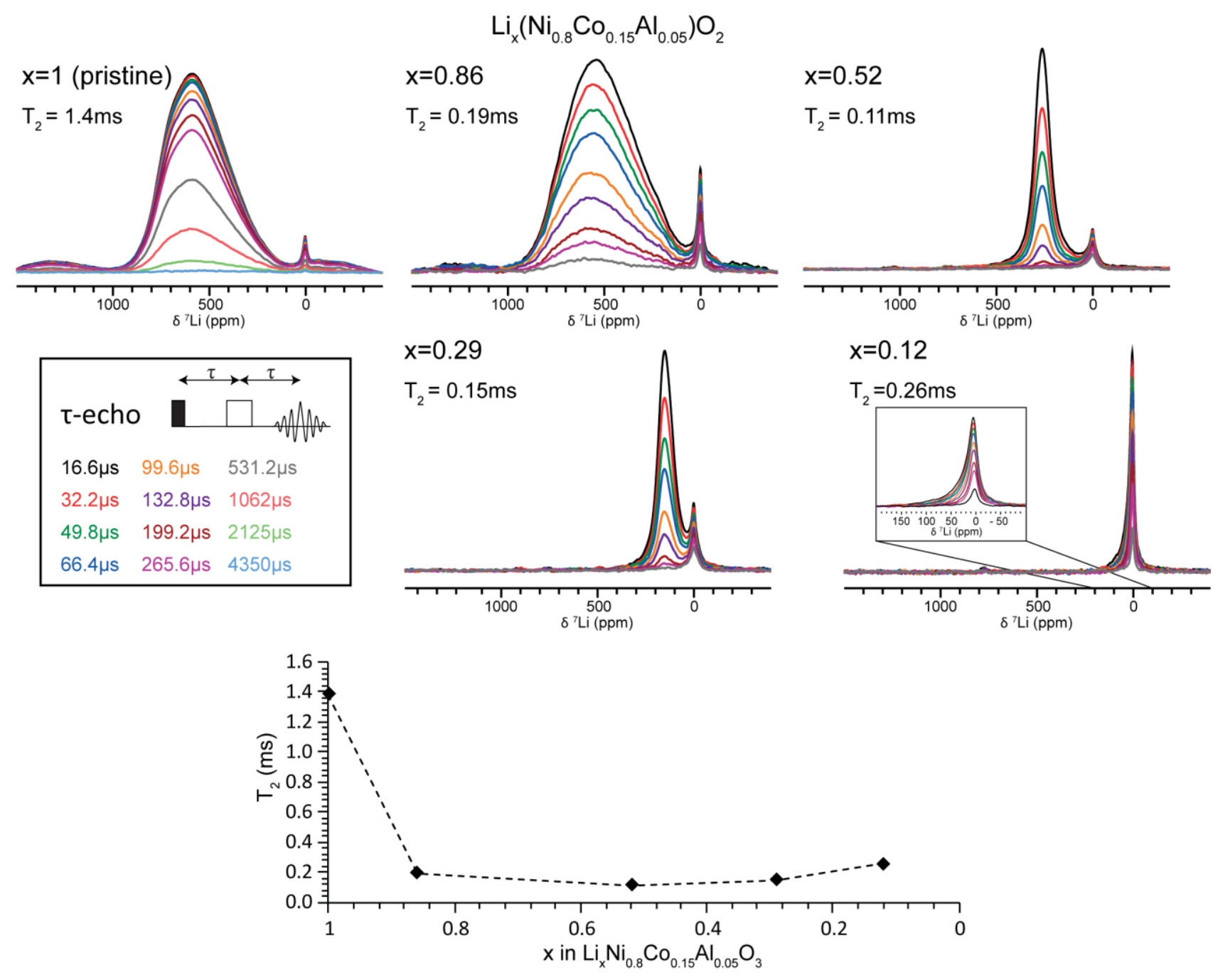

Figure S8. ${ }^{7} \mathrm{Li}$ NMR spectra for HT-TODA-NCA samples at various states of charge with variable echo delays. The $\mathrm{T}_{2} \mathrm{~S}$ for each sample are listed. The ratio of the intensity observed after the minimum echo delay $\left(I_{33 \mu s}\right)$ versus the theoretical intensity at 0 echo delay $\left(I_{0 \mu s}\right)$ is plotted. This plot shows the trend in the $T_{2} S$ with shorter $T_{2}$ giving lower $I_{33 \mu s} / I_{0 \mu s}$ values. The lower $T_{2}$ observed for the $x=0.86$ sample may indicate that, although no motional averaging is observed, an increase in the Li mobility is most likely occurring even in this sample. The $T_{2}$ is lowest for the $x=0.52$ sample which exhibits a fast motion lineshape but still contains a significant concentration of paramagnetic $\mathrm{Ni}^{3+}$. The slight increase observed on further charging may be caused by a reduction in the mobility but will also be affected by the decreasing number of paramagnetic centres, which cause fast relaxation. Spectra were recorded using Hahn-echo pulse sequences at $60 \mathrm{kHz}$ using a Bruker 1.3mm MAS probe at 4.7 T. $\mathrm{T}_{2} \mathrm{~S}$ were measured by iterating the length of the Hahn--echo delay ( $\mathbf{T}$ ) and modelling the loss of signal intensity using an exponential decay function. The Hahn-echo pulse sequences were rotor-synchronised. Hence, all delays are multiples of the rotational period $16.6 \mu \mathrm{s}$.

\section{Modeling of NMR Spectra:}

As outlined in detail in previous publications, ${ }^{5}$ a "random solution" approach can be used to model the spectra of pristine NCA. Briefly, to calculate the NMR spectrum that would result for a random solution of cations, all possible Li environments that can occur in NCA were enumerated (no antisite disorder was considered), then each was assigned a probability based on the stoichiometry using the multinomial formula. The shift was then calculated by summing the Fermi-contact shift contribution for each neighbor-as Fermi-contact shifts are, to a good approximation, additive. ${ }^{7}$ The NMR shift depends strongly on whether the bond pathway goes via a long or short Jahn-Teller (JT) axis, ${ }^{7}$ however as the JT axes are dynamic on the NMR timescale ${ }^{5}$ the shifts were assumed to be averaged. The Fermi contact shifts in this work were assumed to be $-15 \mathrm{ppm}$ and $125 \mathrm{ppm}$ for 
$\mathrm{Ni}^{3+}-\mathrm{O}-\mathrm{Li}\left(90^{\circ}\right)$ and $\mathrm{Ni}^{3+}-\mathrm{O}-\mathrm{Li}\left(180^{\circ}\right)$ respectively in line with the values calculated by Middlemiss et al. ${ }^{7}$ and observed experimentally for $\mathrm{Li}\left[\mathrm{Ni}_{x} \mathrm{Mn}_{\mathrm{x}} \mathrm{Co}_{(1-2 \mathrm{x})}\right] \mathrm{O}_{2}$ by $\mathrm{Zeng}$ et al. ${ }^{8} \mathrm{Co}^{4+}$ shifts were not considered.

However, once the NCA enters the fast Li motion regime, the broad spectrum predicted by the statistical model becomes insufficient. In order to model the lineshape of the mobile Li, the calculated probabilities and shifts were converted into chemical exchange lineshapes using Norris' formula as outlined previously ${ }^{9,10}$. In order to make use of this convenient formula the native linewidth for a single, non-overlapping, non-exchanging, Li environment $\left(v_{1 / 2, \text { no-exch }}\right)$ must be determined. In addition, $v_{1 / 2, \text { no-exch }}$ is assumed to be the same for every Li environment. As $v_{1 / 2, n o-e x c h}$ cannot be measured in this case a sensible approximation must be used. The linewidth of another paramagnetic compound, without multiple Li environments could be used to estimate $v_{1 / 2, \text { no-exch }}$. However, any paramagnetic compound will have a linewidth induced by temperature gradients in the rotor and even a seemingly ideal reference like layered $\mathrm{LiNiO}_{2}$ has a dynamically averaged JT distortion which will lead to broadening. Layered $\mathrm{LiMnO}_{2}$, could be a good reference, however as well as containing a different paramagnetic ion it's static, cooperative JT distortion will give rise to a large electron-nuclear dipolar anisotropy, again broadening the signal. The linewidth of the 0ppm diamagnetic peak, present in all the samples in this work, are $1000-2000 \mathrm{~Hz}$; these peaks are likely a composite of several different diamagnetic Li environments but provide a reasonable reference point. Considering this, using a $v_{1 / 2, n o-e x c h}$ in the region of $1000 \mathrm{~Hz}$ is reasonable. For an upper bound, the sharpest peak modelled in this work $\left(\mathrm{x}=0.52\right.$ at $\left.77^{\circ} \mathrm{C}\right)$, which has a linewidth of ca. $5000 \mathrm{~Hz}$ cannot be modelled with a $v_{1 / 2, \text { no-exch }}$ greater than $2300 \mathrm{~Hz}$, even if an infinite of the hopping rate is chosen. From these considerations, values of 500,1000 and $1500 \mathrm{~Hz}$ were used to calculate hopping rates to illustrate the trends in Li mobility in NCA.

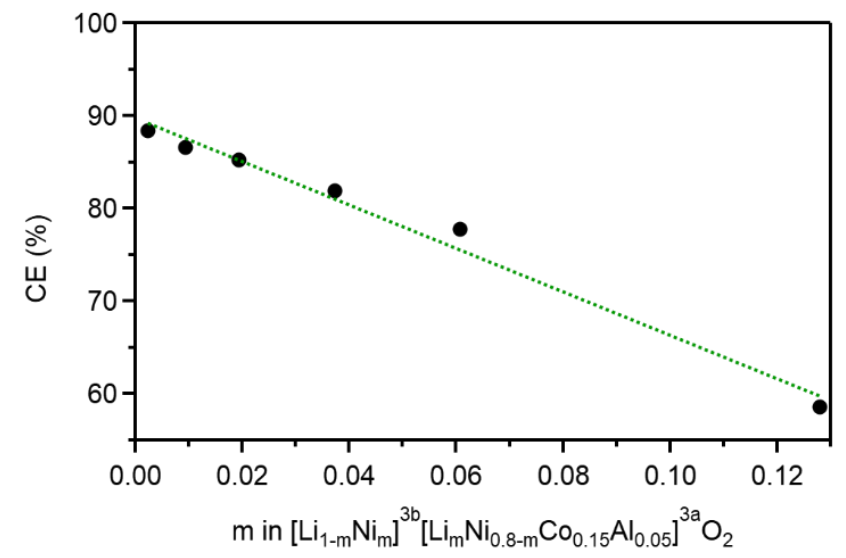

Figure S9. First-galvanostatic-cycle $\left(2.5-4.2 \mathrm{~V}\right.$ at $\mathrm{C} / 10$, at $\left.30^{\circ} \mathrm{C}\right)$ Coulombic efficiency of NCA prepared with increasing amount of interlayer mixing. The dashed line represents a linear fit of the data. Values were taken from the work of Makimura et al. ${ }^{11}$ They achieved varying amounts of interlayer mixing by varying the ratio of $\mathrm{Li}$ to $\mathrm{TM}$ ratio during synthesis. 


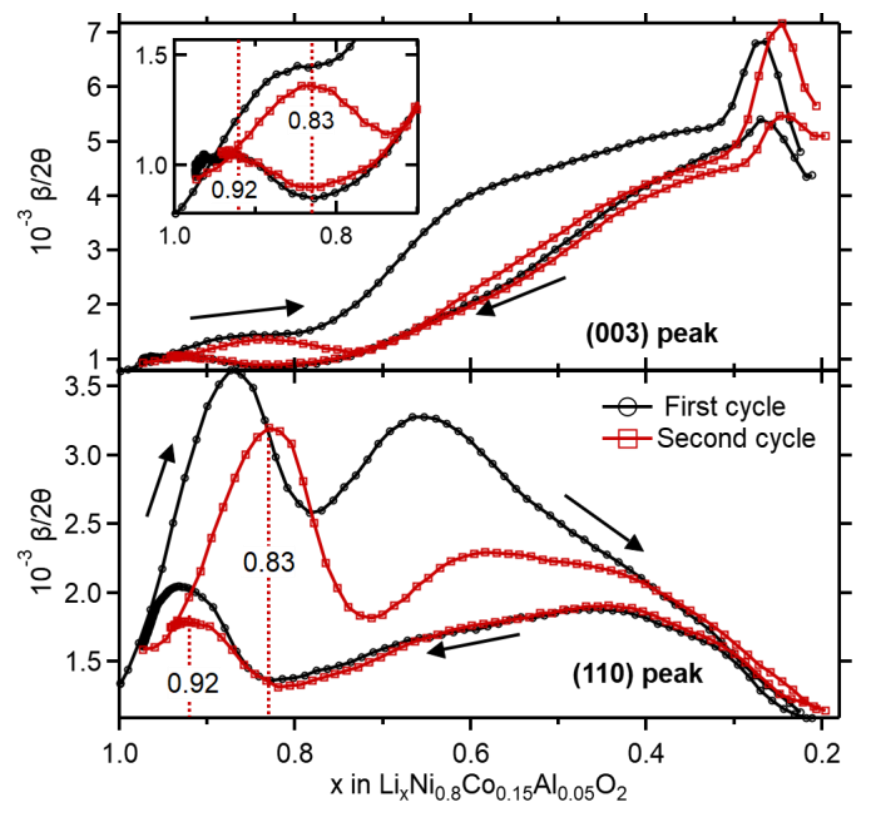

Figure S10. Variation of the net peak broadening $\beta$ (full width at half maximum minus instrument contributions) divided by the peak position $2 \theta$ for (003) and (110) peaks obtained from the operando XRD data recorded on NCA. We represent here the net increase of peak width obtained from the fit, divided by the $2 \theta$ position of the peak to circumvent issues related to the $2 \theta$-dependance of the broadening.

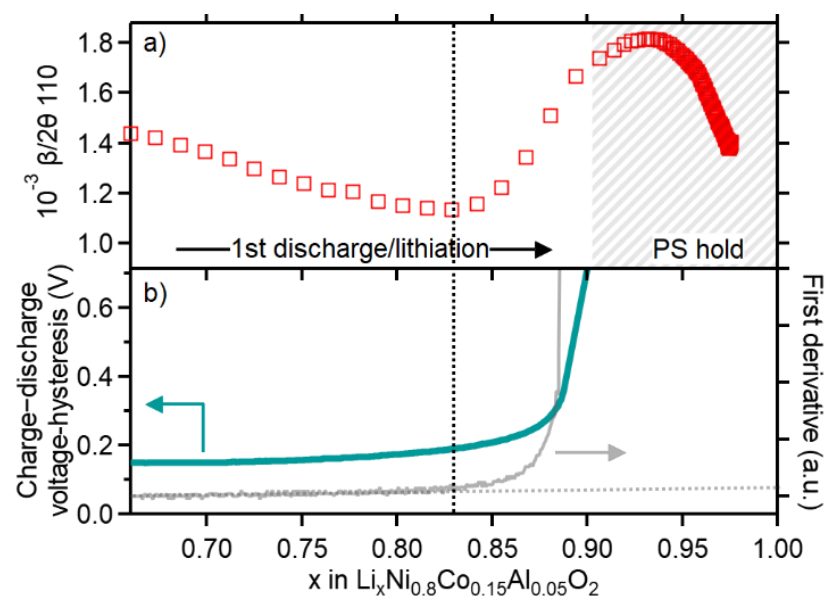

Figure S11. (a) Variation of the net peak broadening $\beta$ (full width at half maximum minus instrument contributions) divided by the peak position $2 \theta$ for (110) peak. The shaded area denotes the potentiostatic hold. (b) Charge-discharge voltage hysteresis (blue) and its corresponding first derivative (grey). The horizontal dashed line on panel $b$ is an extension of the near-linear rate of change of the voltage hysteresis in the $x=0.66-0.75$ range. The vertical dashed line is a guide for the eye.

\section{Anisotropic peak broadening in NCA:}

Observed peak broadening (Figure S10) reflects both Li compositional heterogeneity (concentration gradient) and structural disorder (inhomogeneous strains also called microstrains ${ }^{12,13}$, stacking faults, dislocations ${ }^{14}$ ). Broadening present during charge is mainly induced by the reaction heterogeneity: the two populations are fit by a single, broader peak. The broadening observed during discharge, however, can be ascribed to increasing structural disorder. This broadening is anisotropic in nature. The width of the (110) peak, which reflects disorder within the layers, increases more than that of the (003) peak, which reflects disorder between layers. Note, we represent here the net (minus instrumental contributions) increase of peak width (denoted $\beta$ ) obtained 
from the fit, divided by the $2 \theta$ position of the peak to circumvent issues related to the $2 \theta$-dependance of the broadening. We argue that the anisotropic broadening during discharge is likely induced by microstrains because size effects are negligible. ${ }^{12,13}$ Microstrains increase significantly in the high $x$ regime $(1>x>0.8)$. During the second delithation, microstrains attains a maximum value at $x=0.83\left(1 / 6^{\text {th }} \mathrm{Li}\right)$ and spans $(1>x>0.7)$. During lithation, the increase in microstrains attains a maximum value at $x=0.92\left(1 / 12^{\text {th }} \mathrm{Li}\right)$ and and spans $(1>x>0.83)$. Similar trends, albeit to different extent, are observed for $\varepsilon$ along [110] and [003] directions, which reflect increased amount of microstrains within the layers and between layers, respectively.

On discharge, the start of the slow Li-diffusion regime can be observed via the rapid increase of the charge-discharge voltage hysteresis at $x>0.85$ (Figure S11). This matches the onset of the anisotropic broadening of XRD peaks which we attribute to disorder within the layers. While charge-discharge voltage hysteresis continuously increases as lithiation proceeds, the rate at which it increases (i.e., the derivative) accelerates as $x$ increases in the $0.85<x<0.92$ range. While the Jahn-Teller distortion in both LNO and NCA is known to be dynamic, ${ }^{5,15}$ there may be thermodynamically preferred orderings which control the average structure. For example, certain pairwise orientations of $\mathrm{Ni}^{3+} \mathrm{JT}$ axes have been calculated to be favorable. ${ }^{16}$ The relaxation of the microstrains below $\sim 0.92 \mathrm{Li}$ may therefore correspond to a $\mathrm{Ni}^{3+}$ concentration threshold, beyond which a less frustrated, lower energy average structure is adopted. This relaxation is not associated with an increase in Li transport as the vacancy concentration decreases and tends to zero. This phenomenon requires further investigation.

\section{High-voltage increase of $R_{w p}(x<0.3)$ :}

The high-voltage $(x<0.3)$ increase of the $R_{w p}(<2 \%$ at $x=0.3$ to $\sim 3.5 \%$ at $x \approx 0.21)$ observed in Figure $3 e$ can be attributed to peak asymmetry caused by a distribution in $x$. Figure $\mathbf{S 1 2}$ shows how two 003 peaks are necessary to fit the XRD profile at the top of charge $(x \approx 0.21)$, when $R_{w p}$ is at its maximum, while a single peak is sufficient to fit the pristine data.
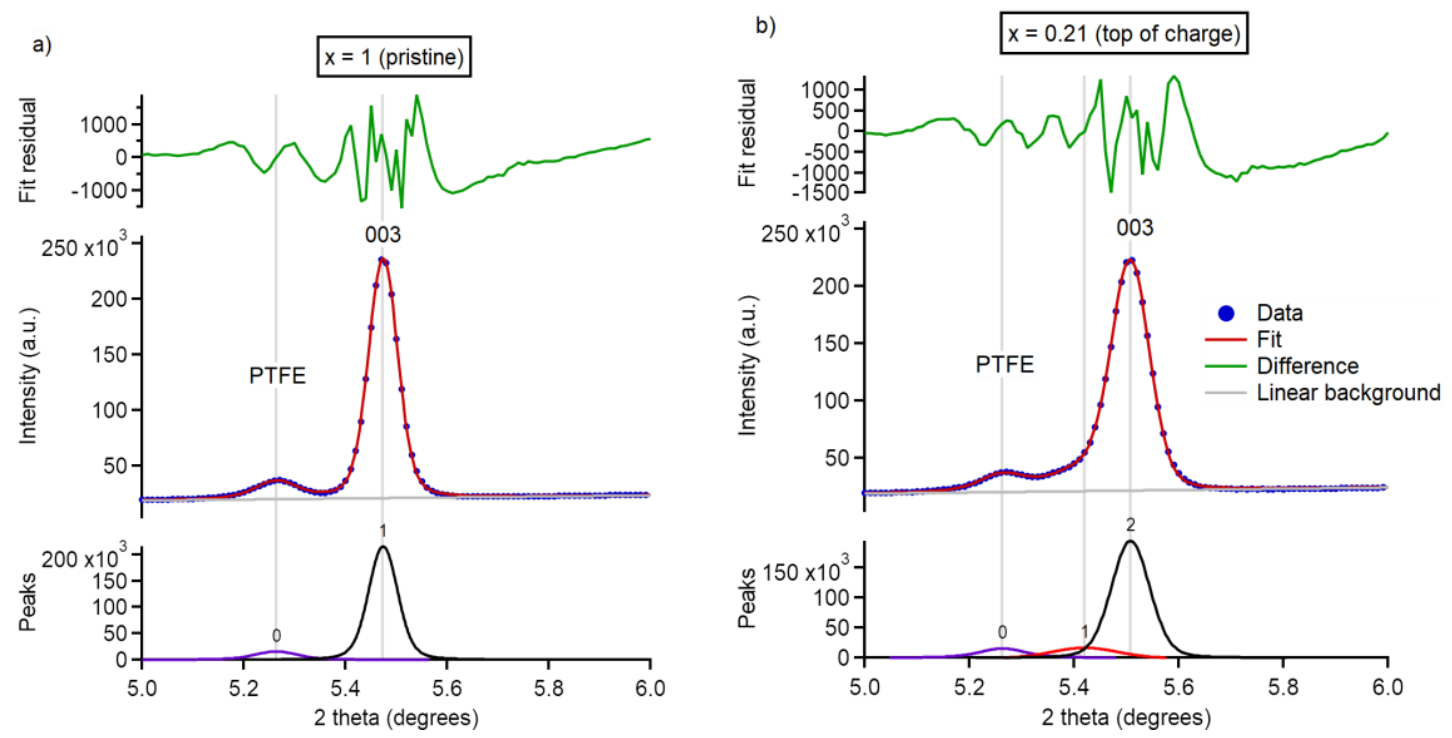

Figure S12. Multipeak fits (Voigt profiles) of the XRD data $(\lambda=0.4526 \AA$ ) collected a) before cycling $(X=1)$, and $b)$ at the top of the first charge $(x=0.21)$. Peak at $\sim 5.26^{\circ}$ is from the polytetrafluoroethylene powder used as binder in the electrode. In the pristine NCA, a single 003 peak is sufficient to obtain a good fit while two 003 peaks are necessary to obtain a good fit at the top of charge.

A two-phase Rietveld refinement for $x=0.21$, following the same protocol used to obtain Figure 4 , decreases the $R_{w p}$ to $2.29 \%$ (Figure S13). Considering the value of the refined $c$ lattice parameter in the additional phase $(14.463 \AA$ ), it seems that the additional NCA population (NCA2; $\sim 6.4$ wt. \%) is more lithiated ( $x=0.28$ estimated based on $c$ lattice parameter) than the main/initial NCA population (NCA1) and therefore appears to be delithiated more slowly (Table S3). This in contrast with the socalled $\mathrm{H} 2-\mathrm{H} 3$ phase transition observed for $\mathrm{LiNiO}_{2},{ }^{17}$ where the additional phase $\mathrm{H} 3$ has a significantly smaller $c$ lattice parameter than the initial phase $\mathrm{H} 2$. This observation suggests a kinetic limitation in NCA, which is somewhat surprising considering the persisting motional narrowing of ${ }^{7} \mathrm{Li}$ NMR lineshape at $x=0.12$ (Figure S8). The narrow lineshape suggests that NCA is still in the fast Li motion regime in that $x$ range. More work will be needed to understand this phenomenon. 


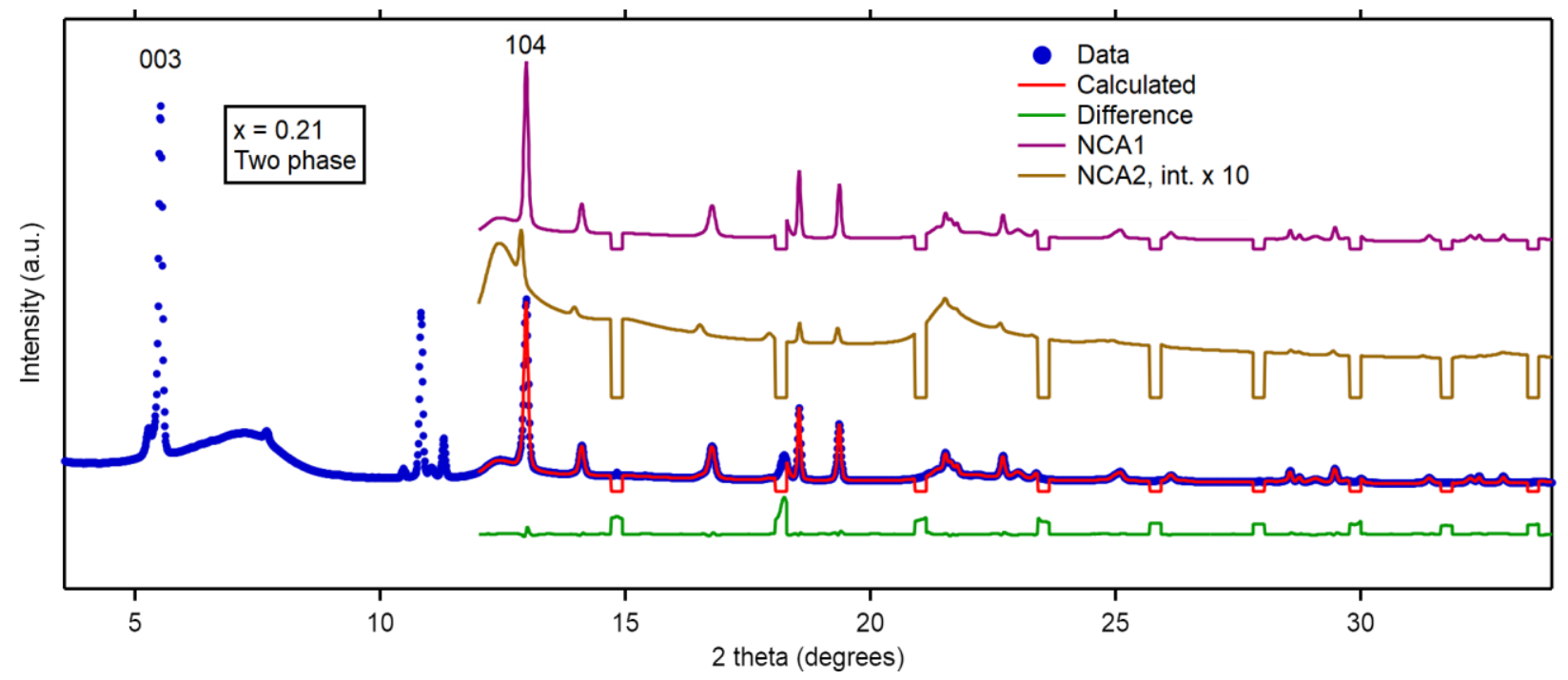

Figure S13. Two-phase (both $R-3 \mathrm{~m})$ Rietveld refinement to the operando XRD $(\lambda=0.4526 \AA)$ data recorded on NCA at $x=0.21$, in the first charge. The contribution of each phase to the fit is shown. The intensity contribution from the additional phase, NCA2, was multiplied by 10 for added clarity. $R_{\text {wp }}=2.29 \%$.

Table S3. Structural parameters obtained from the two-phase Rietveld ( $R-3 m$ space group) refinement to the operando XRD record on NCA at $x=0.21$ shown Figure S13.

\begin{tabular}{|l|c|c|}
\hline & NCA1 & NCA2 \\
\hline a lattice parameter $(\dot{A})$ & $2.80701(3)$ & $2.8060(4)$ \\
\hline c lattice parameter $(\dot{A})$ & $14.1095(4)$ & $14.463(5)$ \\
\hline O $z$ coordinate & $0.2644(1)$ & $0.264(1)$ \\
\hline Phase fraction (wt. \%) & $93.6(1)$ & $6.4(1)$ \\
\hline$x$ estimated from $c$ lattice parameter & $\mathrm{N} / \mathrm{A},<0.21$ & 0.28 \\
\hline
\end{tabular}

References

(1) Saadoune, I.; Delmas, C. $\mathrm{LiNi}_{1-y} \mathrm{Co}_{y} \mathrm{O}_{2}$ Positive Electrode Materials: Relationships between the Structure, Physical Properties and Electrochemical Behaviour. J. Mater. Chem. 1996, 6 (2), 193-199.

(2) Saadoune, I.; Ménétrier, M.; Delmas, C. Redox Processes in $\mathrm{Li}_{x} \mathrm{Ni}_{1-y} \mathrm{Co}_{y} \mathrm{O}_{2}$ Cobalt-Rich Phases. J. Mater. Chem. 1997, 7 (12), 25052511.

(3) Kobayashi, H.; Shigemura, H.; Tabuchi, M.; Sakaebe, H. Electrochemical Properties of Hydrothermally Obtained $\mathrm{LiCo}_{1-x} \mathrm{Fe}_{\mathrm{x}} \mathrm{O}_{2}$ as a Positive Electrode Material for Rechargeable Lithium Batteries. J. Electrochem. Soc. 2000, 147 (3), 960-969.

(4) Ménétrier, M.; Shao-Horn, Y.; Wattiaux, A.; Fournès, L.; Delmas, C. Iron Substitution in Lithium-Overstoichiometric "Li 1.1 CoO 2 ": Combined 57 Fe Mössbauer and 7 Li NMR Spectroscopies Studies. Chem. Mater. 2005, 17 (18), 4653-4659.

(5) Trease, N. M.; Seymour, I. D.; Radin, M. D.; Liu, H.; Liu, H.; Hy, S.; Chernova, N.; Parikh, P.; Devaraj, A.; Wiaderek, K. M.; Chupas, P. J.; Chapman, K. W.; Whittingham, M. S.; Meng, Y. S.; Van Der Van, A.; Grey, C. P. Identifying the Distribution of $\mathrm{Al}^{3+}$ in $\mathrm{LiNi}_{0.8} \mathrm{Co}_{0.15} \mathrm{Al}_{0.05} \mathrm{O}_{2}$. Chem. Mater. 2016, 28 (22), 8170-8180.

(6) Carlier, D.; Ménétrier, M.; Grey, C.; Delmas, C.; Ceder, G. Understanding the NMR Shifts in Paramagnetic Transition Metal Oxides Using Density Functional Theory Calculations. Phys. Rev. B 2003, 67, 1-14.

(7) Middlemiss, D. S.; llott, A. J.; Clément, R. J.; Strobridge, F. C.; Grey, C. P. Density Functional Theory-Based Bond Pathway Decompositions of Hyperfine Shifts: Equipping Solid-State NMR to Characterize Atomic Environments in Paramagnetic Materials. Chem. Mater. 2013, 25 (9), 1723-1734. 
(8) Zeng, D.; Cabana, J.; Yoon, W.-S.; Grey, C. P. Investigation of the Structural Changes in $\mathrm{Li}^{2}\left[\mathrm{Ni}_{\mathrm{y}} \mathrm{Mn}_{\mathrm{y}} \mathrm{Co}(1-2 \mathrm{y})\right] \mathrm{O}_{2}(y=0.05)$ upon Electrochemical Lithium Deintercalation †. Chem. Mater. 2010, 22 (3), 1209-1219.

(9) Norris, J. R. Rapid Computation of Magnetic Resonance Line Shapes for Exchange among Many Sites. Chem. Phys. Lett. 1967, 1 (8), 333-334.

(10) Märker, K.; Reeves, P. J.; Xu, C.; Griffith, K. J.; Grey, C. P. Evolution of Structure and Lithium Dynamics in $\mathrm{LiNi}_{0.8} \mathrm{Mn}_{0.1} \mathrm{Co}_{0.1} \mathrm{O}_{2}$ (NMC811) Cathodes during Electrochemical Cycling. Chem. Mater. 2019, 31 (7), 2545-2554.

(11) Makimura, Y.; Sasaki, T.; Nonaka, T.; Nishimura, Y. F.; Uyama, T.; Okuda, C.; Itou, Y.; Takeuchi, Y. Factors Affecting Cycling Life of $\mathrm{LiNi}_{0.8} \mathrm{Co}_{0.15} \mathrm{Al}_{0.05} \mathrm{O}_{2}$ for Lithium-Ion Batteries. J. Mater. Chem. A 2016, No. 4, 8350-8358.

(12) Robert, R.; Novák, P. Switch of the Charge Storage Mechanism of $\mathrm{Li}_{x} \mathrm{Ni}_{0.8} \mathrm{Co}_{0.15} \mathrm{Al}_{0.05} \mathrm{O}_{2}$ at Overdischarge Conditions. Chem. Mater. 2018, 30 (6), 1907-1911.

(13) Robert, R.; Novak, P. Structural Changes and Microstrain Generated on $\mathrm{LiNi}_{0.8} \mathrm{Co}_{0.15} \mathrm{Al}_{0.05} \mathrm{O}_{2}$ during Cycling: Effects on the Electrochemical Performance. J. Electrochem. Soc. 2015, 162 (9), A1823-A1828.

(14) Singer, A.; Hy, S.; Zhang, M.; Cela, D.; Fang, C.; Qiu, B.; Xia, Y.; Liu, Z.; Ulvestad, A.; Hua, N.; Wingert, J.; Liu, H.; Sprung, M.; Zozulya, A. V.; Maxey, E.; Harder, R.; Meng, Y. S.; Shpyrko, O. G.; Hy, S.; Cela, D.; Fang, C.; Wynn, T. A.; Qiu, B.; Xia, Y.; Liu, Z.; Ulvestad, A.; Hua, N.; Wingert, J.; Liu, H.; Sprung, M.; Zozulya, A. V.; Maxey, E.; Harder, R.; Meng, Y. S.; Shpyrko, O. G. Nucleation of Dislocations and Their Dynamics in Layered Oxides Cathode Materials during Battery Charging. Nat. Energy 2017, 3 (August), 17.

(15) Rougier, A.; Delmas, C.; Chadwick, A. V. Non-Cooperative Jahn-Teller Effect in LiNiO 2 : An EXAFS Study. Solid State Commun. 1995, $94(2), 123-127$.

(16) Vernay, F.; Penc, K.; Fazekas, P.; Mila, F. Orbital Degeneracy as a Source of Frustration in $\mathrm{LiNiO}_{2}$. Phys. Rev. B 2004, 70 (1), 014428.

(17) Li, H.; Zhang, N.; Li, J.; Dahn, J. R. Updating the Structure and Electrochemistry of $\mathrm{Li}_{x} \mathrm{NiO}_{2}$ for $0 \leq x \leq 1$. J. Electrochem. Soc. 2018, 165 (13), A2985-A2993. 